\title{
IL COMPORTAMENTO DI SUOLI AGRARI RIGONFIABILI
}

\author{
Luigi Cavazza, Adriano Guarnieri, Antonia Patruno, Elio Cirillo
}

\section{INTRODUZIONE}

\section{Introduction}

Notevoli sono gli sviluppi teorici, anche a scala submicroscopica e microscopica, riguardanti il processo di rigonfiamento e contrazione dei suoli, nonché quelli a scala più ampia a carattere sia ingegneristico che agronomico, soprattutto a carattere più applicativo. Una sintesi di queste conoscenze sembra opportuna. In questa nota ci si limita ad illustrare alcuni aspetti tecnici ed applicativi alla scala geotecnica tanto ingegneristica che, si potrebbe dire agronomica.

Si danno qui per scontate le nozioni di base su cui si fondano le manifestazioni di contrazione e rigonfiamento [48] ed i suoi limiti [12,15], e si sposta l'attenzione sulle più aggiornate rappresentazioni di questo fenomeno a livello di caratterizzazione dei materiali ed alla loro trattazione a fini applicativi soprattutto in relazione agli aspetti idrologici. Qui di seguito si farà riferimento alla terminologia più generale da tempo proposta dall'UNI-ISO $^{(1)}$ [17] e nello specifico per i suoli rigonfiabili, alcuni termini più diffusi tra gli Autori anglofoni ma anche alcune opportune proposte recenti degli Autori francesi, come sarà indicato di volta in volta. Si noti, incidentalmente, come col passare dall'esame di materiali rigidi a quelli rigonfiabili diventa molto spesso preferibile, anche per fini idrologici, il ri-

Paper received 19.12.2006; accepted 15.10.2007

Prof. L. CAVAZZA, Professore emerito Università di Bologna, già ordinario di Agronomia Generale, Dipartimento di Scienze e Tecnologie Agroambientali, DiSTA. Prof.ssa A. PATRUNo, già Professore associato di Fisica del Terreno Agrario, DiSTA, stessa Università. Prof. A. GUARNIERI, Professore ordinario Dipartimento Economia e Ingegneria Agrarie, DEIAGRA, stessa Università. Dott. E. CIRILlo, Assegnista Post-Dottorato di Ricerca, DiSTA, stessa Università.

1 Soprattutto i termini "massico" e "volumico" nel senso di "per unità di massa" e "per unità di volume". Si useranno inoltre simboli scelti come più facilmente intuibili per il lettore italiano. In particolare $\rho_{\text {aps }}=$ massa volumica apparente dei solidi $\left(\rho_{\mathrm{b}}\right.$, soil dry bulk density in inglese); $\rho_{\mathrm{s}}=$ massa volumica reale dei solidi; $\rho_{1}=$ massa volumica dell' acqua; $w=$ umidità gravimetrica $\left(\mathrm{kg} \mathrm{kg}^{-1}\right)=\vartheta \rho_{\mathrm{l}} / \rho_{\mathrm{s}}$ con $\vartheta=$ rapporto di umidità; $V_{\text {map }}=$ volume massico apparente $\left(=\rho_{\mathrm{aps}}^{-1}\right), V_{\mathrm{s}}$ bulk specific volume, in inglese. ferimento al volume del materiale solido anziché alla massa del solido o volume complessivo del materiale (criteri di rapporto del vuoto e rapporto di umidità). È frequente oggi sostituire alla massa volumica apparente il volume massico apparente $\left(V_{\text {map }}=\rho_{\text {aps }}{ }^{-1}\right)$ soprattutto per la facilità di confronto con le variabili comuni tra $\mathrm{i}$ geotecnici (p.es. relazioni $V_{\text {map }}(w)$ invece della $e(\vartheta)$; fig. 1).

\section{CONSIDERAZIONI SULLA CURVA DI CONTRAZIONE}

\section{Shrinkage curve}

In passato, una buona parte delle ricerche era effettuata su argilla, eventualmente estratta dai terreni (pasta di argilla) o su terreno finemente setacciato; (fig 2a). Lo studio riportato a livello di terreno, cioè su materiale più o meno strutturato e contenente anche limo e sabbia, ha richiesto modifiche e generalizzazioni della stessa curva di contrazione, pur restando l'argilla l'agente fondamentale del rigonfiamento, e ciò malgrado una notevole variabilità da un caso all'altro.

Partendo dalla saturazione di un terreno si è reso necessario prendere in considerazione un' ulteriore porzione della curva di contrazione, normalmente assente nelle curve su pasta di argilla e che tenesse conto dell'acqua contenuta nei macropori di questa porzione. Si cominci con l'esaminare la curva di contrazione caratteristica di un terreno agrario rigonfiabile cominciando dal suo stato di massimo rigonfiamento; per i casi di dispersione indefinita (possibili per suoli fortemente alcalinizzati e che qui non si considerano) si ricordino i criteri di base [12].

Secondo le proposte di Stirk [42] a seguito di perdita graduale dell'umidità a partire da un campione di terreno saturo nelle condizioni in situ ha inizio un campo di variazione, che indicheremo come dominio $^{(2)}$ di umidità strutturale, durante il quale la perdita

2 È il termine corretto usato da Boivin et al., [5] in sostituzione del più comune termine di "fase". 


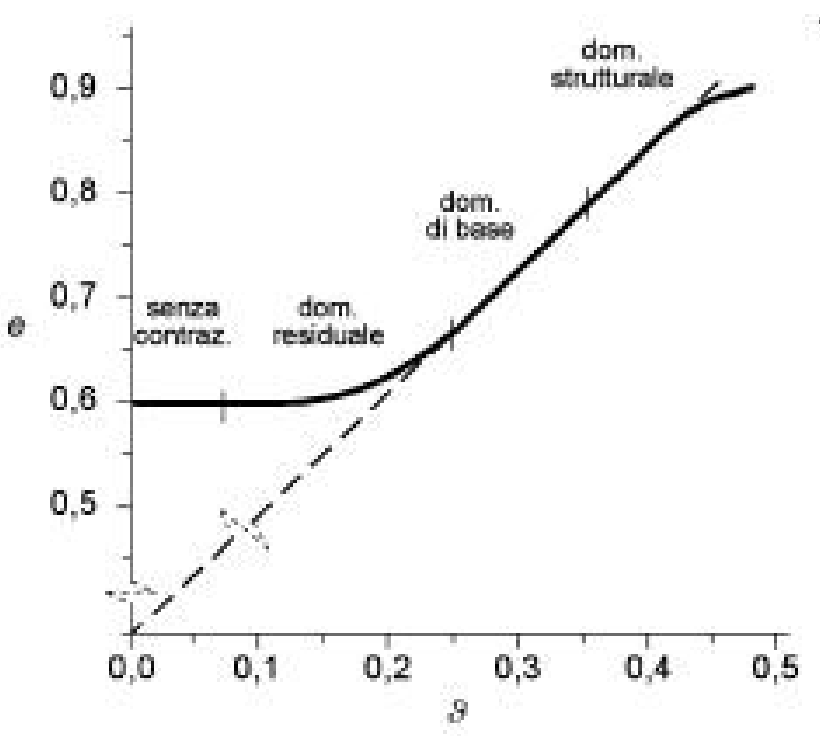

Fig. 1 - Relazione tra la funzione $e(\vartheta)$ e quella $V_{\text {map }}(w)$.

Fig. 1 - Relationship between $\mathrm{e}(\vartheta)$ and $\mathrm{V}_{\mathrm{s}}(\mathrm{w})$ functions.

di acqua in volume, spesso irregolare, è in genere superiore alla riduzione del volume del suolo $(\mathrm{d} w>$ $\mathrm{d} V_{\text {map }}$; ciò in generale implica pendenza media della curva di contrazione $<1$ ). Il contributo di questo dominio al contenuto idrico del terreno può raggiungere anche il $60-80 \%$ di quello totale (fig. 2b). Il processo di contrazione dipende fortemente dalla struttura del terreno e dalla stabilità di questa (concetto ancora poco considerato). Esso è generalmente esposto a maggiore variabilità spaziale e verosimilmente anche temporale (per effetto di instabilità strutturale). È un dominio che ancora oggi, anche se di massimo interesse agronomico, resta poco studiato [10]. In esso spesso è mal distinto lo spazio che occupano le fessure del suolo, tra cui alcuni Autori distinguono gli spazi interpedali (tra i "ped"(3)) da quelli intrapedali (o macropori di vario tipo e forma; fig. 2e). In generale in condizioni di prosciugamento l'entrata d'aria in questi pori è pressoché immediata.

Col proseguire del prosciugamento si passa al $d o-$ minio di contrazione "di base" (in inglese: basic; in passato detto normale) ${ }^{(4)}$ che è quello classicamente studiato sulle paste di argilla, oggetto ancora oggi di ricerche fondamentali; è caratterizzato da perdita di acqua ( $w$, assumendo $\rho_{1}=1$ ) esattamente eguale alla perdita in volume del terreno $\left(\Delta V_{\text {map }}=\Delta w\right.$ perciò $\mathrm{d} V$ $\left.{ }_{\text {map }} / \mathrm{d} w=1\right)$ segmento rettilineo sulla cosiddetta "retta di carico" o di saturazione $e(\vartheta)$, e tale che il terreno

3 I ped sono unità strutturali naturali, le cui dimensioni possono cambiare con l'umidità, separate dai ped adiacenti a mezzo di superfici virtuali di minore resistenza meccanica, o di rivestimenti vari, o da fessure [31].

4 Il termine normale è ancora largamente usato dai ricercatori Anglofoni . La sua sostituzione è stata proposta per unificazione della terminologia con i geotecnici da Braudeau e Touma [6] col termine "principale". Nel 1992, però, Mitchell e VanGenuchten [32] e successivamente Boivin et al. [5] hanno utilizzato il termine di "dominio di base", che qui useremo.

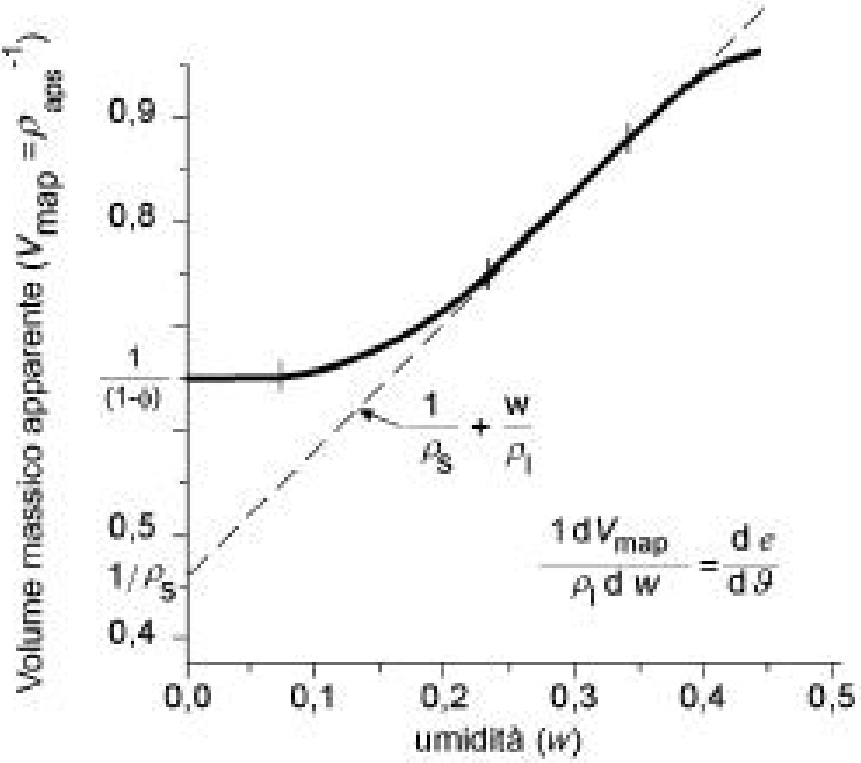

resta saturo. Entro tale dominio la riduzione di acqua può lasciare decrescere il suo potenziale matriciale anche al di sotto dei $-1500 \mathrm{kPa}$, convenzionale coefficiente di avvizzimento [49, 4].

Se si considera il terreno e non la pasta di argilla, i micropori che caratterizzano questo dominio sono ancora completamente saturi di acqua, ma l'aria e qualche frazione di acqua residua contenuta nei macropori del dominio strutturale fanno sì che il rapporto $\mathrm{d} V_{\text {map }} / \mathrm{d} w$ non sia più pari ad 1 , come nella pasta di argilla (vari in fig. 2). Tenendo conto dei macropori svuotatisi nella fase strutturale, sono spesso le porzioni rimanenti del suolo che restano sature e si tratta perciò di stato di saturazione degli aggregati distintisi nel corso del precedente prosciugamento (p.es. grumi) . Si noti che se si trattasse di terreni rigidi, la perdita di acqua in questo dominio comporterebbe la sostituzione con aria dell'acqua persa e tale dominio non avrebbe senso; è la possibilità di contrazione del suolo rigonfiabile che può assicurare la condizione $\Delta V_{\text {map }}=$ $\Delta w$ e spiega l'interesse particolare di questo dominio nei suoli rigonfiabili [28].

Se prosegue il prosciugamento, la curva di contrazione $V_{\text {op }}(w)$ si allontana dalla "retta di carico" o non è più parallela a questa $\mathrm{e}$, anche per la pasta di argilla; si ha $\Delta w>\Delta V_{\text {map }}$ e perciò l'acqua persa è sostituita da aria anche nei micropori e, data la natura rigonfiabile del terreno, ha pressoché contemporaneamente inizio la fessurazione. Il passaggio a questa condizione detta "dominio residuale" (fig. 2, vari) è indicato come punto di ingresso di "entrata d'aria" nei micropori. Questo punto può verificarsi, secondo il tipo di terreno, in corrispondenza di un qualsiasi valore della retta di carico e la curva che rappresenta questo dominio residuale ha concavità verso l'alto. Per questo e per il dominio successivo assumono interesse le considerazioni riguardanti la teoria dello "stato critico" del suolo. 

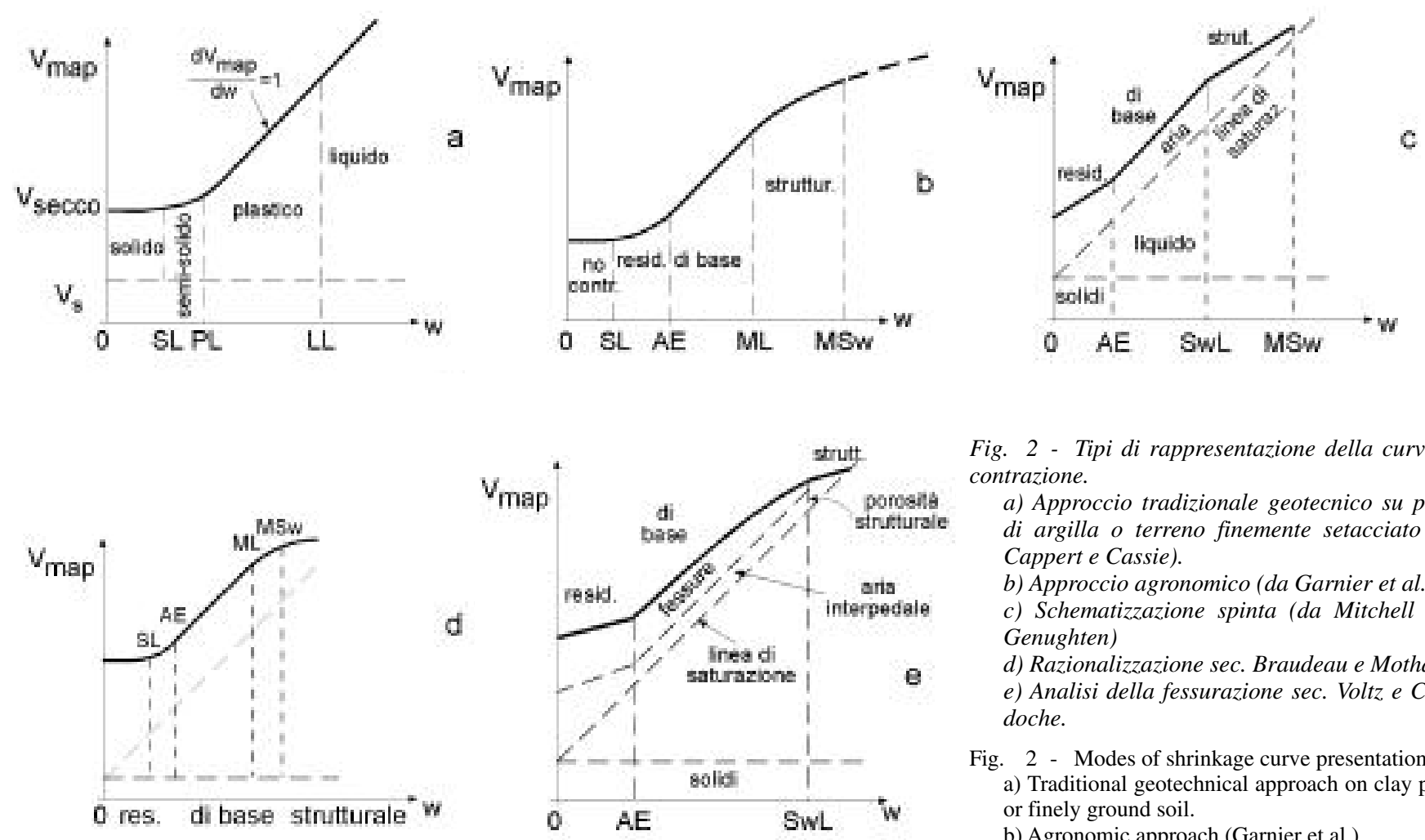

Fig. 2 - Tipi di rappresentazione della curva di contrazione.

a) Approccio tradizionale geotecnico su pasta di argilla o terreno finemente setacciato (da Cappert e Cassie).

b) Approccio agronomico (da Garnier et al.).

c) Schematizzazione spinta (da Mitchell e v. Genughten)

d) Razionalizzazione sec. Braudeau e Mothar.

e) Analisi della fessurazione sec. Voltz e Cabidoche.

Fig. 2 - Modes of shrinkage curve presentation. a) Traditional geotechnical approach on clay paste or finely ground soil.

b) Agronomic approach (Garnier et al.)

c) Linearized schema (Mitchell and v. Genughten)

d) Rationalized (Braudeau e Mothar)

e) Analysis of cracking (Voltz and Cabidoche)

Segue, nello schema tradizionale, un dominio di contrazione nulla con $\Delta V_{\text {map }}=0$ al variare di $w$ (fig. b). In alcune trattazioni teoriche [25] questo dominio è assorbito da quello precedente (ne rappresenta il limite).

Nel complesso per l'intera curva di contrazione sono note varianti diverse soprattutto come schematizzazioni dei domini mediante segmenti rettilinei contigui $[30,22,47,7]$ eventualmente collegati da archi di cui si danno equazioni empiriche.

Gli aspetti teorici più approfonditi sono quelli di Groenevelt e Bolt [25] che considerano l'intera contrazione solo come insieme di dominio "di base" e "residuale". Una notevole applicazione recente ai problemi agronomici è stata fatta da Groenevelt et al. [26], tuttavia con le stesse limitazioni di Groenevelt e Bolt [25]. Un notevole progresso di questi approfondimenti portano alla stima del cosiddetto potenziale di sovraccarico $(\Omega)$, che resta tuttavia ancora uno dei problemi applicativi di più difficile soluzione.

Per quanto riguarda l'applicazione a concrete condizioni di campo la trattazione di Cabidoche e Ruy [10] prende in più seria considerazione il ruolo del "dominio strutturale" come più fondamentale proprio nei vertisuoli in coltura.

Frequente in tutte queste ricerche è il ricorso al termine di "matrice" o "matrice argillosa", inteso come porzione del materiale terroso delimitato da fessure ed il cui volume è perciò complementare con quello di queste ultime $[41,19,16]$; questi termini sono, peraltro, pure utilizzati nel senso di suolo inizialmente saturo [8]. Recentemente Boivin et al. [5] hanno proposto in tal senso il termine "plasma", che qui useremo.

In realtà tutta la precedente schematizzazione trascura, quando non ignora, la ben nota possibilità di frammentazione gerarchica della struttura del suolo [33] che complica notevolmente lo schema sopra esposto ma rende meglio conto di quello più articolato ottenibile nei casi concreti; vi si avvicina la trattazione di Chertkov [16].

\section{LE RELAZIONI TRA CURVA DI RITENZIONE IDRICA E CURVA DI CONTRAZIONE}

\section{Relationship between retention curve and shrinkage curve}

In tutto quanto esposto sin qui l'attenzione alle relazioni tra $V_{\text {map }}$, contenuto gravimetrico di acqua nel terreno $(w)$ e le condizioni di carico meccanico sul suolo, ha finito col trascurare le relazioni col potenziale dell'acqua. Varie ricerche avevano messo in evidenza l'analogia formale tra la curva di ritenzione del terreno per l'acqua (water retention curve; WRC) e le curve di contrazione (shrinkage curve; ShC), essendo entrambe funzione dell'umidità. Un esame molto accurato delle 
relazioni tra contrazioni dei terreni e stato dell' acqua nel loro sistema porale è stato effettuato da Boivin, Garnier e Tessier [4]. Essi confermano che nello stato aggregato si ha, per il dominio di base, contrazione come con la pasta di argilla, anche se con rapporto $\mathrm{d} V_{\text {map }} / \mathrm{d} w$ diverso da 1 , in relazione alla struttura ed alla stabilità di questa; la porosità nel dominio saturo (di base) è ora costituita sia dai micropori del plasma che come porosità interna dei microaggregati di argilla. Yule e Ritchie [49] avevano già segnalato potenziali matriciali di circa $-1500 \mathrm{kPa}$ per umidità comprese entro il dominio di base; dalle ricerche di Boivin [3] emerge in particolare che l'acqua all'estremo più umido del dominio saturo si trova ad un potenziale matriciale di circa $-10 \mathrm{kPa}$ (è quindi vicino alla convenzionale capacità idrica di campo), mentre si avvicina a $-1500 \mathrm{kPa}$ (il convenzionale coefficiente di avvizzimento) non lontano dall' altro suo estremo, in vicinanza del punto di entrata d' aria. Nel dominio di base sarebbe quindi compresa quasi tutta l'acqua disponibile nel senso tradizionale (si tornerà su questo concetto al termine di questo lavoro). In questo dominio il gradiente $\mathrm{d} V_{\text {map }} / \mathrm{d} w$ cresce notevolmente col diminuire del diametro dei pori e soprattutto col crescere del contenuto di argilla del terreno (mentre varia poco col crescere dei diametri entro il dominio strutturale).

Un recente studio molto accurato di Boivin, Garnier e Vauclin [5] ha affrontato su un'ampia serie di tipi pedologici e contenuto di argilla (dal 3,5 al 65\%), sui terreni sia indisturbati (carote), che setacciati, un'ampia sperimentazione in proposito, confrontando valori tensiometrici (nel campo della tensiometria) e variazioni volumiche dei materiali. Questi Autori hanno effettuato i confronti utilizzando per i vari materiali i parametri di Van Genuchten [45] di WRC, nella forma che prevede la valutazione dell'umidità in peso (w) e, per $V_{\text {map, }}$ una modifica di detta equazione inizialmente proposta da Peng e Horn [35] che esprime la curva di contrazione in funzione della stessa umidità. I risultati sono stati in generale sorprendentemente soddisfacenti con solo poche discordanze tra $\mathrm{i}$ due tipi di curve (WRC e ShC) e ciò soprattutto in corrispondenza delle porzioni lineari ed agli estremi della curva di contrazione (fig. 2d); particolarmente coincidenti sono i valori di umidità delle due curve nei punti di transizione della curva di contrazione, almeno utilizzando per questa il modello di Braudeau e Mohtar ([7]; mod. XP; fig. 2d). Questi Autori ritengono di potere dedurre la WRC, di determinazione lunga e brigosa, dalle curve di contrazione, di più comoda determinazione.

In realtà, se si considera la flessibilità intrinseca dell'equazione di Van Genuchten in contrasto con la rigida schematicità (per tratti lineari) del modello di Braudeau per i vari domini della curva di contrazione, sembra quasi ovvio che, specialmente nella porzione centrale lineare dei domini di Braudeau, la WRC debba scostarsi un po' verso l'alto. Resta però degno di particolare attenzione il dominio di base che dovrebbe essere rettilineo e quindi incompatibile con la WRC.
Si rilevi, infatti, che in questo dominio c'è saturazione e quindi il potenziale matriciale dell'acqua ai suoi estremi deve essere tale che in esso il raggio dei micropori, cioè la curvatura media dell'interfaccia liquido-aria, sia:

$$
r \leq-2 \gamma / \psi_{\mathrm{pm}}
$$

in cui $\gamma$ è la tensione superficiale della fase liquida e $\psi_{\mathrm{pm}}$ è il potenziale matriciale volumico corrispondente all'umidità $(w)$ del terreno p. es. ai due estremi del dominio. Dallo schema della fig. 3 si deduce che al variare dell'umidità $w$ in questo dominio, varia pure il rapporto del vuoto $(e)$, che si contrae linearmente da un estremo all'altro di $(w)$, perciò spreme in un certo modo l'acqua dai pori di cui riduce $\mathrm{i}$ diametri. All'interno dei pori deve perciò variare anche la pressione intergranulare, cioè la pressione di rigonfiamento $(\Pi)$ tra le particelle che compongono la massa solida e si deve avere:

$$
\psi_{\mathrm{pm}, \mathrm{mis}}=+\Pi+\psi_{\mathrm{pm} \text {,int }}
$$

con $\psi_{\mathrm{pm} \text {,mis }}$ potenziale misurato per l' acqua nel terreno, e $\psi_{\text {pm,int }}$ il potenziale dell' acqua vigente nei pori (essendo $\Pi>0$ e $\psi_{\text {pm,mis }}$ e $\psi_{\text {pm,int }}<0$ ). C'è perciò una pressione nella fase liquida interna al poro additiva con il $\Pi$ (in accordo con Kim et al., 1999) la quale cambia forse anche con la distanza dalle pareti solide. Non si hanno criteri sicuri per dire come cambi $\Pi(w)$, $\mathrm{ma}$ in generale questa funzione non è rettilinea (quando aumenta il volume della fase liquida nel poro c'è

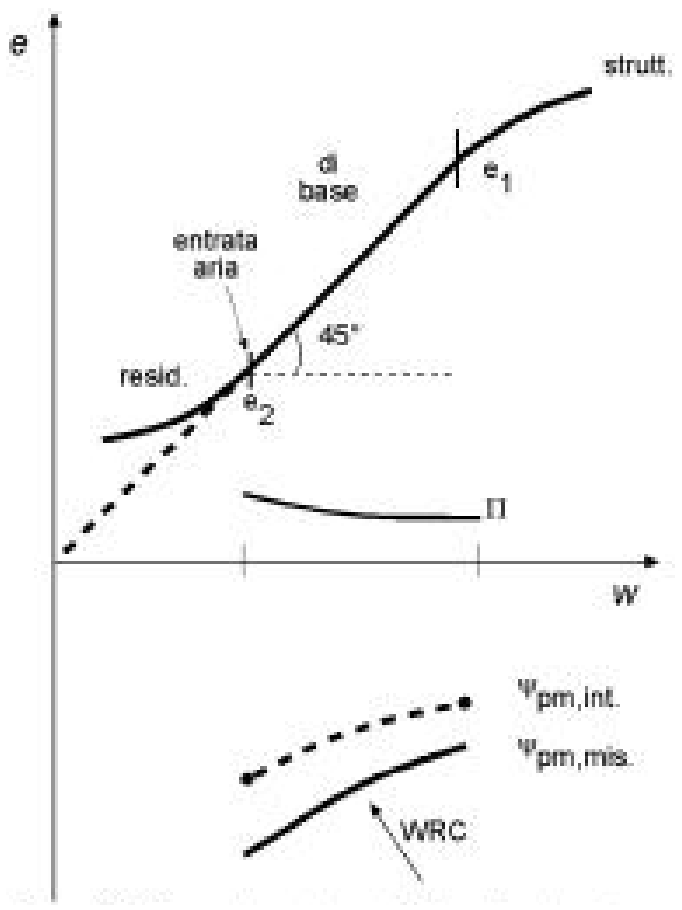

Fig. 3 - Schematizzazione della relazione tra curva di contrazione $(S h C)$ e variazione di e in relazione alla curva di ritenzione idrica (WRC) per il dominio di base. $\Pi=$ pressione di rigonfiamento o intergranulare.

Fig. 3 - Schema of the relationship between ShC and consequence of the WRC curves in the basic domain. $\Pi=$ swelling pressure; $\psi_{\mathrm{pm}, \mathrm{mis}}, \psi_{\mathrm{pm} \text {,int }}=$ measurable and internal pore pressure. 
diminuzione del $\Pi$, e ciò tende ad attenuarsi col procedere verso il rigonfiamento massimo del terreno). Ciò significa che alla contrazione di $e$, che accompagna la riduzione di $w$, corrisponde una riduzione non lineare di $\psi_{\mathrm{pm}, \text { int }}<\psi_{\mathrm{pm} \text {,mis }}$, che rende conto dell'inconciliabile discordanza tra WRC e ShC per questo dominio (a parte possibilità di accettabile approssimazione nelle condizioni pratiche). Forse più accurate determinazioni potrebbero scaturire dalle variazioni di scala [2].

\section{IL POTENZIALE DI SOVRACCARICO}

\section{Overburden potential}

Quando si prendono in considerazione condizioni di pieno campo (si tratta ora di suolo in senso pedologico e non di terreno) i problemi diventano più complessi, anche indipendentemente dalla loro coltura che ne può complicare lo studio [10].

Innanzitutto deve essere considerato che col crescere della profondità (supponendo che non vi sia sovraccarico alla superficie del suolo) il peso stesso del terreno, sovrastante un certo livello, esercita una pressione su quello sottostante, che dipende fortemente tanto dal materiale solido quanto dal grado di saturazione in acqua dei vari strati sovrastanti. Se il suolo fosse secco, il carico totale ad ogni profondità sarebbe sopportato dalla parte solida e dato da tale carico totale su quel piano; se fosse perfettamente saturo d'acqua, tutto il carico sarebbe sopportato dall'acqua. Il problema sorge per ogni umidità intermedia e col suo variare lungo il profilo. In tal caso, del carico totale (pari alla sommatoria delle masse volumiche umide lungo tutto il sovrastante profilo) solo una parte incide sul potenziale dell'acqua al livello considerato e precisamente si ha che il potenziale tensiometrico misurabile ad ogni data profondità è dato [38] da:

$$
\Phi=\psi_{\mathrm{pm}}+\alpha \underset{\mathrm{z}}{0} \rho_{\text {apu }} g d z
$$

in cui $\Phi$ è il potenziale che si può leggere su un tensiometro inserito alla profondità considerata; $\psi_{\mathrm{pm}}$ è il potenziale che per quella stessa umidità si troverebbe sulla curva WRC di quel terreno determinata senza carico con le comuni tecniche; $\rho_{\text {apu }}$ è la massa volumica umida del suolo alle varie sovrastanti profondità; $g$ è l'accelerazione di gravità; $\alpha$ è il coefficiente di carico esprimente la frazione del carico totale sopportata dall'acqua. In pratica $\alpha=0$ in terreno secco; $\alpha=1$ in terreno saturo; mentre $\alpha$ varia per ogni suolo e per ogni distribuzione dell'acqua lungo il sovrastante profilo negli altri casi intermedi. Quest'ultima constatazione rende la determinazione dell' $\alpha$, nelle varie condizioni pratiche, talmente problematica e variabile nel tempo, che quasi sempre è trascurata dai tecnici. ( $\mathrm{Si}$ ricordi l'equivalenza tra i grafici di $e(\vartheta)$ e di $V_{\text {map }}(w)$ come in fig. 1.

Le conseguenze pratiche delle possibili variazioni dell' $\alpha$ sono in realtà notevoli e, per il comune modo

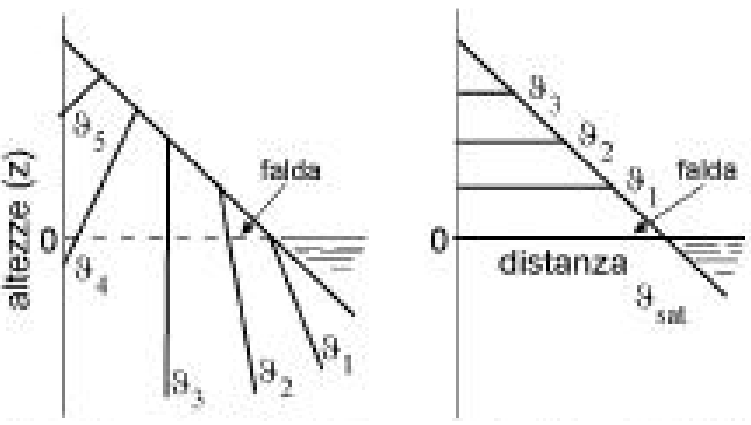

Fig. 4 - Comportamento dell'acqua nei suoli rigonfiabili (in sinistra) rispetto a quelli rigidi (in destra). Da Philip [38].

Fig. 4 - Behaviour of water in swelling soil (left) or in rigid soil (right) (from Philip [38]).

di interpretare i processi idrologici del suolo, sconvolgenti (fig. 4). Per casi particolari sono note tecniche idonee a mettere in evidenza la loro importanza [44, 14]; una formula proposta da Groenevelt e Bolt [25] non è facilmente applicabile.

Va segnalato che mentre questo genere di problemi ha importanza rilevante per la statica e la dinamica dell'acqua entro lo strato di alcuni metri di profondità (p.es. per problemi di drenaggio) in assenza di piante; la presenza di queste altera per lo più tanto l'ordine di grandezza dei potenziali coinvolti, sì che chi si occupa dell'utilizzazione dell'acqua da parte delle piante trova la loro importanza, non sempre opportunamente, secondaria.

\section{IL PROBLEMA DELLA FESSURAZIONE}

\section{Cracking problems}

In pieno campo gli strati superficiali del suolo, anche se incolto, sono soggetti alle vicende meteoriche che vi fanno variare continuamente e notevolmente certe caratteristiche fisiche, pur con più o meno graduale transizione col procedere in profondità.

Innanzitutto, sia ad un esame ad ampia scala territoriale che anche a scala di dettaglio, se lo strato umido non è ancora fessurato, la più opportuna direzione dei movimenti da considerare è quella unidimensionale, verticale (1D) con evidenti conseguenze sulla subsidenza del suolo. In pratica lo stato del suolo, in assenza di piante, è dominato dal gradiente di umidità imposto dalle vicende meteoriche. Perciò, mentre possono restare unidimensionali gli strati saturi inferiori, in quelli superiori possono insorgere fessurazioni che rendono possibile alle sue unità strutturali, p.es. i ped, un' espansione tridimensionale (3D) entro le fessure. Lo studio può indurre allora a considerare il comportamento del suolo sia nel suo insieme che invece in dettaglio, quando avviene su suoi campioni naturalmente definiti (i singoli ped) o opportunamente prelevati (p.es. carote ottenute con l'inserimento di cilindri nel suolo) su cui successivamente si esaminano particolari aspetti (variazioni tridimensionali del volume, 
tipo di fessurazioni interne, formazione di vie preferenziali per flussi liquidi e trasporti salini, ecc.; [19]).

Problematico ne risulta in pratica il confronto tra situazioni a contrazione-rigonfiamento tridimensionali (3D), con quelle di casi unidimensionali, 1D (p.es problema delle variazioni di subsidenza di superficie, relativamente ampia e non di singoli "ped"), come pure nel confronto della variazione da $3 \mathrm{D}$ in campo ed 1D, fornita da edometri.

Il passaggio della dimensione è stato affrontato da Bronswijk, [8], che, ignorando la fase strutturale, ha proposto la formula:

$$
1-\frac{\Delta V}{V}=\left(1-\frac{\Delta z}{z}\right)^{6}
$$

in cui $V$ è il volume originario del terreno saturo; $z$ è lo spessore di un cubo del detto volume $V$, mentre $r_{\mathrm{s}}$ è un fattore adimensionale che permette di convertire valori osservati di cambiamenti verticali di spessore (1D) in cambiamenti tridimensionali (3D) di volume (collega la subsidenza al volume totale nello strato in contrazione). Nel caso di subsidenza $(\Delta z>0)$ senza fessurazione (caso 1D) si ha $r_{\mathrm{s}}=1$; nel caso di fessurazione senza subsidenza $(\Delta z=0)$ e si avrebbe $r_{\mathrm{s}} \rightarrow \infty$. Per casi in cui si abbia contemporaneamente subsidenza e fessurazione, si avrebbe $r_{\mathrm{s}}=3$ se la contrazione fosse isotropica, $1<r_{\mathrm{s}}<3$ se domina la subsidenza, e $r_{\mathrm{s}}>3$ se domina l'effetto di fessurazione. La formula è stata verificata da Bronswick [8] anche per i casi di suoli sottoposti a diversi potenziali di sovraccarico, $\Omega$, e perciò a diversa profondità.

Secondo un successivo schema [9] le variazioni $\Delta V$ vengono espresse per unità di area rigonfiabile misurata alla superficie del suolo e perciò volumi di plasma, volumi di fessure e volumi di acqua risultano espressi in altezze anziché in volumi $\left(\mathrm{m}\right.$ anziché $\left.\mathrm{m}^{3}\right) \mathrm{e}$ la (4a) diventa perciò:

$$
\Delta V=\left[1-\left(1-\frac{\Delta z}{z}\right)^{r} \cdot\right] z
$$

Questa formula, con qualche semplificazione introdotta da Yule e Ritchie [49], portò Bronswijk all'equazione più generale:

$$
\Delta \mathrm{W}=\mathrm{S}+\Delta \mathrm{V}+\frac{[\mathrm{c}(9)-9]}{1+\mathrm{c}_{\mathrm{s}}}
$$

valida per tutto il campo della contrazione, inclusa perciò la fase strutturale, $S$ (questa è tale che $S+\Delta V$ della eq. 5 , opportunamente espressa, sia pari a $\Delta V$ della $4 b$ ) ed incluse pure le fasi residue e a zero contrazione, nonché i casi di contrazione anisotropica. Nella 5 si ha $e=$ rapporto del vuoto; $\vartheta=$ rapporto di umidità; $e_{\mathrm{s}}=$ rapporto del vuoto in condizioni di saturazione; l'ultima frazione nel membro di destra della 5 rappresenta la parte di acqua contenuta nelle fasi residuali e senza contrazione; $r_{\mathrm{s}}$ delle eq. $4 \mathrm{a}$ e $4 \mathrm{~b}$ tiene conto di eventuale anisotropia. Nella precedente relazione si assume: a) che $\Delta w=\Delta V$; b) che le variazioni di $z$ implichino variazioni di spessore e perciò che non vi siano o siano trascurabili le fessure orizzontali; c) che entro la fase strutturale sia $\Delta V=0$ (si vedrà in seguito che ciò può non essere sempre accettato).

Alle fondamentali relazioni introdotte da Bronswijk, vanno poi aggiunte le considerazioni di Chertkov [16] secondo cui ulteriori precisazioni sono necessarie. I criteri di Bronswijk, infatti non tengono ancora conto a) dello stato di tensione (stretching) a cui il terreno saturo è sottoposto quando è in fase di perdita di acqua e perciò di contrazione (ed a cui si deve il successivo incipiente cedimento che porta alla fessurazione $)^{(5)}$ b) che anche nei campioni prelevati per lo studio in campo, quando sono in essiccamento, possono formarsi fessure; c) che non c'è motivo per assumere che il fattore $r_{\mathrm{s}}$, nel corso dell'essiccamento, non sia funzione dell'umidità.

Chertkov, perciò, su basi sperimentali (dati suoi di laboratorio e dati di Hallaire; [27], su suolo strutturato, in campo), introduce il coefficiente di elasticità di Poisson nei calcoli, giungendo alla seguente formulazione di sintesi per l' $r_{\mathrm{s}}$ corretto di Bronswijk $\left(r_{\mathrm{s} \mathrm{cor}}\right)$ :

$$
r_{\text {scox }}(w)=M(w) \times L(w) \times r_{s}(w)
$$

in cui $r_{\mathrm{s}}(w)$ è il valore di Bronswijk reso funzione dell'umidità; $M(w)$ e $L(w)$ sono funzioni miranti a correggere l' $r_{\mathrm{s}}$ secondo Chertkov e precisamente:

$$
L(\mathrm{w})=\frac{\log \left[\bar{V}_{1}(w) / \bar{V}_{0}\right]}{\log \left\{x[\mu(w)]^{2} V_{1}^{\prime}(w) \bar{V}_{0}\right\}}
$$

e $M$ è dedotto da:

$$
\bar{V} / \bar{V}_{0}=\left(\bar{V}_{\cdot} / \bar{V}_{0}\right)^{M}
$$

essendo nelle (6abc): $\bar{V}=$ volume iniziale dello strato nel modello Bronswijk; $\bar{V}_{0}=V_{\text {map }}$ iniziale del plasma; $\bar{V}_{s}=V_{\text {map }}$ del campione con fessure, $\bar{V}_{1}=V_{\text {map }}$ di strato fessurato sec. Bronswijk;

Inoltre si ha che:

$$
x(\mu)=(\mu+1 / 4)^{1 / 2}-1 / 2
$$

con

$$
\mu=2 p /(1-p)
$$

ove $v$ è il rapporto di Poisson del plasma senza fessure.

Si noti ancora che per terreno aggregato si può ottenere:

$$
\chi=\left[\left(\mathrm{e}_{1}+1\right) /\left(\mathrm{e}_{1}^{\prime}+1\right)\right]^{1 / 2}
$$

da cui si ricava $\chi$ e poi $\mu$ e $v$.

L'aspetto fondamentale di questo approccio è che si presuppongono determinazioni varie $\left(\bar{V}_{1} ; \bar{V}_{\mathrm{s}} ; V / \bar{V}_{\mathrm{s}}\right)$ da effettuare sperimentalmente in funzione dell'umidità nel corso del prosciugamento. I risultati che si ottengono sono significativamente diversi da quelli otte-

5 È una manifestazione troppo spesso trascurata, ma importante quando ci si occupa degli effetti sulle radici coinvolte in questi processi. 
nuti con la sola applicazione di Bronswijk. Si noti che coi criteri di Chertkov si possono affrontare anche $i$ casi di suoli strutturati.

Molte ricerche sulle relazioni di rigonfiamento-umidità del suolo sono effettuate operando su campioni prelevati in campo, sia come prelevamento di volume noto (mediante inserimento di cilindri metallici; [19]) che come esame di un singolo ped [31]. Fondamentale è in tali casi la possibilità di rilevare al variare dell'umidità, tanto le variazioni verticali quanto quelle orizzontali del campione in esame. Diventa importante esaminare la relazione tra il comportamento della zolla o del campione in esame e quello dell'intero suolo in campo (sui campioni si sogliono però determinare le contrazioni e non i rigonfiamenti, che implicherebbero considerazioni sulle fessure esterne ai campioni).

Se si considera l'acqua nel terreno intorno a $\psi=$ $1500 \mathrm{kPa}$, ci si trova ancora in condizioni di "dominio di base". In tal caso si trova, teoricamente e sperimentalmente $[1,49]$ che la perdita $\Delta w$ di acqua (in termini di altezza, entro tutto lo strato considerato) corrisponde in prima approssimazione [31] a :

$$
\Delta w=3 \Delta s(1+\Delta s / z)
$$

in cui $\Delta s$ è la contrazione verticale conseguente alla perdita di acqua $\Delta w$ da tutto lo strato di terreno $z$. Se il rapporto $\Delta s / z$, come in pratica si verifica, è $<<1$ la precedente può essere approssimata in :

$$
\Delta w / \Delta s=3
$$

Comunque, secondo Yule e Ritchie [49] si ha:

$$
\Delta w=3 \Delta \mathrm{s}(1+\Delta s / z)+W_{\mathrm{s}}
$$

in cui $W_{\mathrm{s}}$ è l'acqua strutturale ( $z$ è preso positivo verso l'alto). Dalla 9 a e per $\Delta s / z<<1$ si deduce l'interessante relazione:

$$
W_{\mathrm{s}} / \Delta s=\Delta w / \Delta s-3
$$

Mc Intyre [31] in particolare propose come meccanismo di contrazione pedale, partendo dalla soppressione della maggiore quantità di acqua strutturale e assumendo che il prosciugamento di un ped avvenga con sola formazione di fessurazione verticali, che l'acqua che il ped perde è solo $1 / 3$ della quantità di acqua che complessivamente perde il suolo nel suo complesso, restando impegnata la rimanente acqua per riempire $\mathrm{i}$ vuoti creatisi tra i vari ped, i quali si restringono. L'aumento del $\rho_{\text {aps }}$ del ped conseguente a questa sola fessurazione verticale risulterebbe perciò conseguenza della sola acqua persa dal ped che resta ancora in condizioni di dominio di base" e questa acqua può essere solo $1 / 3$ di quella persa complessivamente dal suolo. Se in questo campo di variazione dell'umidità media del suolo nel suo insieme $w$ si applicassero le equazioni $8 \mathrm{a}, 8 \mathrm{~b}, 9 \mathrm{a}, 9 \mathrm{~b}$ ci si imbatterebbe in situazioni anomale trovando $\Delta w / \Delta s=1$ anziché 3 come nella [8b], o meglio, in pratica, risultati intermedi dipendenti essenzialmente da quello che Mc Intyre definisce "grado di pedalità" e dal volume relativo degli altri macropori. Mc Intyre propone di indicare una tale manifestazione come "componente della contrazione apparentemente unidimensionale in dominio di base" dato che la contrazione nelle altre due direzioni non avviene più in questo dominio. Secondo Perroux et. al. [36] si deve parlare in proposito di "componente verticale di una contrazione tridimensionale".

La distribuzione dell' aria tra fessure e porosità intrapedale è considerata da Voltz e Cabidoche [47]; fig. 2e.

Resta degna di ulteriori considerazioni l'interpretazione dell' inverso rigonfiamento di singoli ped o di campioni prelevati con cilindro nell'ambito di un suolo che nel complesso non possa espandersi lateralmente oltre quanto concesso dalle fessure preesistenti.

Si noti qui come spesso nei calcoli richiesti per affrontare i problemi del flusso idrico nei terreni rigonfiabili, che dalle loro deformazioni geometriche, diventa opportuno ricorrere a trasformazioni dei dati, più frequentemente quella del tradizionale sistema euleriano, a quello in coordinate Lagrangiane, o "materiali", o "referenziali", che permettono di affrontare problemi particolari. Importante è la distinzione tra i casi di (a) flusso idrico unidirezionale con deformazione pure verticale unidirezionale (p.es. calcolo del potenziale di sovraccarico e del coefficiente $\alpha$ ); b) flusso idrico unidirezionale con possibilità di deformazione tridimensionale del campione lateralmente non vincolato [24, 29]; c) flusso idrico tridimensionale con deformazione pure tridimensionale, per il quale occorre necessariamente ricorrere al calcolo tensoriale [21].

\section{ASPETTI DINAMICI}

\section{Dynamic aspects}

L'esame sin qui condotto, malgrado le complesse considerazioni riguardanti gli studi dello strato più superficiale dei suoli, concerne prevalentemente condizioni statiche. Sono perciò qui opportune alcune considerazioni sulla conduttività idrica (o conducibilità idraulica) dei suoli rigonfiabili. Varie sono le determinazioni che in proposito si richiedono sia in campo che in laboratorio. Quelle su aggregati naturali (rivestimento con resine idrorepellenti permeabili però al vapor d'acqua) si dimostrano più precise, seppure più laboriose delle determinazioni in condizioni confinate come i cilindretti metallici [18]. Fondamentale é per tutti i terreni la necessità di non limitarsi alla conduttività idrica del terreno saturo, ma di definire la curva della sua variazione in funzione dell'umidità.

$\mathrm{Ci}$ sono almeno due punti fondamentali che distinguono i suoli rigonfiabili: 1) la presenza in uno stesso suolo di domini differenti che possono dar luogo a manifestazioni diverse in relazione all'umidità ed alla distribuzione porale; 2) le conseguenze che sulla conduttività idrica può avere, specialmente in prossimità della superficie del suolo lo stato di rigonfiamento del suolo stesso.

Il primo di questi punti si manifesta essenzialmente in presenza di un dominio strutturale ben sviluppato e 
pone il problema di come affrontare l'eterogeneità del sistema porale nel suo insieme. Da alcuni anni, sotto le esigenze della modellistica ha prevalso per la sua plasticità la rappresentazione della curva di ritenzione per l'acqua (WRC) secondo l'equazione di van Genuchten e Nielsen [46] implicitamente corrispondente ad una distribuzione unimodale dei pori. Più di recente un esame più accurato dei problemi applicativi ha indotto Ross e Smetten [39] a considerare la sovrapposizione di distribuzioni bi o multimodali dei pori in modo da tener conto dei macropori e degli spazi interaggregati, specialmente in vicinanza delle condizioni di saturazione idrica del suolo. Ne è scaturito un criterio molto versatile per ricavarne una funzione composta $K(\psi)$ (scelta per tentativi e con prevalente impiego della stessa funzione di van Genuchten e Nielsen)). Se ne sono ottenuti risultati soddisfacenti in applicazioni concrete di campo [20]. È come tale un risultato di grande rilievo, seppure riferentesi alla determinazione di una costante idrologica senza riferimenti alle proprietà di rigonfiamento del suolo.

Il secondo dei due punti menzionati si riferisce in un certo senso ad una caratteristica più tipica dei suoli rigonfiabili, anche se in pratica il suo effetto può essere mascherato dalla manifestazione precedente. Il fatto è che la conduttività idrica, $K(\vartheta)$ di un suolo rigonfiabile in condizioni insature deve essere definita, in linea di principio, non solo in funzione dell'umidità , $\vartheta$, ma anche di $e$ e, implicitamente, della profondità $z$ [40]. Se si riprende in considerazione la distinzione tra profilo idrico, profilo picnotatico e profilo xerico per suoli saturi (fig. 5) si comprende come in profilo idrico, specialmente nella sua parte superiore, al notevole rigonfiamento corrisponde un aumento della di-

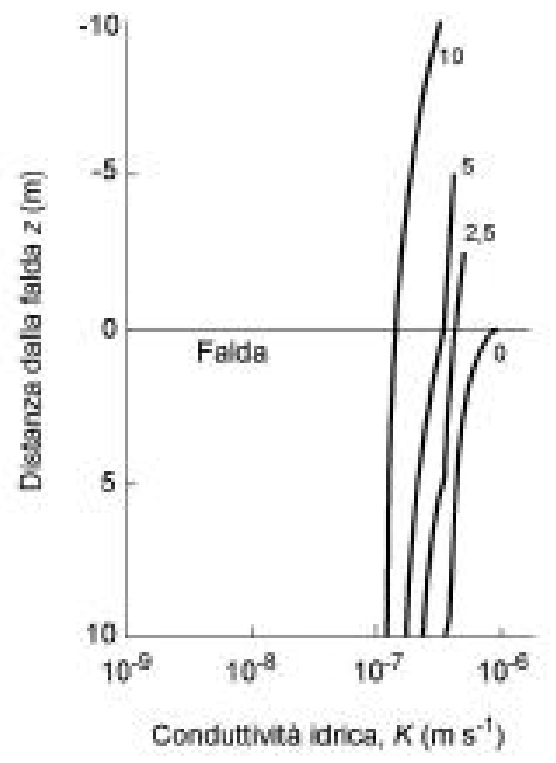

Fig. 5 - Influenza della profondità di falda (numeri sulle curve) sulla conduttività idrica $\left(K_{\mathrm{s}}\right)$ in uno stesso suolo rigonfiabile. Nei suoli non rigonfiabili il $K_{\mathrm{s}} \grave{e}$ unico e costante per tutto il suolo (da Philip [37]).

Fig. 5 - Influence of water table depth (numbers are shown beside the curve) on hydraulic conductivity $\left(K_{\mathrm{s}}\right)$ in the same soil. In non swelling soil $K_{\mathrm{s}}$ is unique and constant for all depths (from Philip [37]). stanza reciproca tra le particelle terrose ossia della porosità e conseguentemente della conduttività idrica. È condizione che può facilmente verificarsi in suoli a struttura instabile con falda alta $\mathrm{o}$, in regime dinamico, nel corso di persistenti piogge che bagnano abbondantemente per un certo tempo la superficie del suolo. È manifestazione di solito trascurata. Dettagli su altre conseguenze verificatesi nel corso di moto stazionario o non stazionario in suoli rigonfiabili uniformi sono schematizzati nei lavori di Philip [38].

\section{L'IMPIEGO DEI CONSOLIDOMETRI}

\section{Consolidometer use}

Un particolare criterio di studio è quello realizzabile mediante l'impiego di consolidometri (o edometri), meglio nella forma di edotensiometri [13]. Sotto vari aspetti questo tipo di approccio è più semplice, ma proprio per questo può risultare più interessante.

Si tratta di prove di infiltrazione unidimensionale che procede generalmente dal basso, in materiale non necessariamente incoerente, di grana o struttura più $o$ meno relativamente uniforme e di caratteristiche note, vincolato lateralmente.

In questo tipo di prove non ci sono in generale problemi di fessurazione, e ovviamente nemmeno di "stretching". Il contenuto idrico medio del campione può essere determinato con cura a mezzo del bilancio idrico; altrettanto dicasi delle relazioni di rigonfiamento-contrazione.

Sono noti due tipi di attrezzature: a) quelle in cui il potenziale di alimentazione dell' acqua al sistema porale è accuratamente monitorato dall'esterno (vari esempi nella letteratura tradizionale; [13]) e b) quelli in cui la pressione porale è monitorata mediante l'utilizzazione di camere a pressione in cui il campione di terreno è immesso per determinarvi il potenziale dell'acqua e l'acqua è ceduta e misurata nella varie condizioni di pressione meccanica applicata al campione di terreno. Col criterio a) ci possono essere difficoltà nel raggiungere grandi potenziali assoluti di alimentazione idrica (è p.es. facile fino a $-200 \mathrm{~cm}$ di potenziale come altezza d'acqua), ma è più difficile il raggiungimento di adeguate masse volumiche apparenti del campione.

Un recente vasto studio di Fritton [21] con l'attrezzatura del tipo b) ha fornito interessanti relazioni quantitative sulla compressione uniassiale di 99 campioni di terreno compresso fino a $2971 \mathrm{kPa}$ e quattro diverse quantità di acqua estrusa, con massa volumica apparente dei campioni fino a $1,8 \mathrm{Mg} \mathrm{m}^{-3}$ ottenendone complesse ma molto interessanti equazioni.

L'esame del materiale a diverse condizioni e differente umidità iniziale fornisce una soddisfacente comprensione dei rapporti tra materiale rigonfiabile e pressioni meccaniche di sovraccarico, così come di potenziale di alimentazione dell'acqua, con possibilità di considerazioni sull'effettiva variazione della "pressione porale" dei campioni in risposta a queste condizioni. 
Più ambizioso ne emerge il desiderio di passare dalle determinazioni con edotensiometro, al constatabile comportamento in campo dei suoli rigonfiabili, il che implica tra l'altro passaggio da 1D a 3D.

\section{CENNI SUL RUOLO DELLE COLTURE}

\section{Hints on cropping effects}

Un rapido cenno merita la constatazione del ruolo che può avere una coltura in relazione alle caratteristiche dei suoli rigonfiabili esplicitamente non affrontato in questa sintesi. Il graduale approfondimento degli apparati radicali e le sue interazioni col regime termopluviometrico del luogo può creare significative differenze nella distribuzione dell' acqua negli strati superficiali dei suoli (p.es. nei $50 \mathrm{~cm}$ superiori) con interessanti differenziazioni di comportamento le quali possono sommarsi al processo di assestamento del suolo inizialmente arato (Cavazza et al. [14]). I criteri di base dello studio della disponibilità dell'acqua in questo tipo di suoli possono essere oggetto di particolari considerazioni [26] anche quando non fanno che aprire nuove problematiche. Sorprendente può apparire la constatazione [10] che, in condizioni ordinarie, tutto il fabbisogno idrico di una coltura può risultare soddisfatto dall'acqua contenuta nel solo dominio strutturale del suolo, senza che le piante attingano da altri domini, anche per difficoltà di trasferimento dell'acqua tra i vari domini. È una condizione ben diversa dal tradizionale modo di concepire l'ordinario ruolo del suolo come serbatoio continuo di acqua per la coltura. È tutto un nuovo approccio nell'esame dei rapporti suolo-coltura per certe condizioni reali e può giustificare un particolare modo di gestione dei vertisuoli [43].

\section{BIBLIOGRAFIA}

\section{References}

[1] Aitchinson G.D., Holmes J.W. 1953. Aspects of swelling in the soil profile. Austr. J. Appl. Sci. 4, 244-259.

[2] BavaYe P., Sposito G. 1984. The operational significance of the continuum hypothesis in the theory of water movement through soils and aquifer. Water Resour. Res. 20, 521-530.

[3] BoIvin P. 1990. Caractérisation physique des sols sulfatés acides de la vallé de Katoure (basse Casamance, Sénegal): Variabilité spatiale et relation avec les caractéristiques pédologique. Ph. Diss. Univ. Paris VI ed ORSTOM, Paris. Serie études et thèse (cit. da Boivin, Garnier, Tessier).

[4] Boivin P., GARnier P., Tessier D. 2004. Relationship between clay content, clay type and shrinkage properties of soil samples. Soil Sci Soc. Am. J. 68, 11451153.

[5] Boivin P., Garnier P., Vauclin M. 2006. Modeling the soil shrinkage and water retention curves with the same equations. Soil Sci. Soc. Am. J. 70, 1082-1093.

[6] Braudeau E., Touma J. 1995. Modeling shrinkage of unconfined soil cores. In Vadose zone hydrology: Cutting across disciplines. Univ. California Davies, 11-12. (Cit. da Garnier, Rieu et al.).

[7] Braudeau E., Mohtar R.H. 2006. Modeling the swelling curve for packed soil aggregates using the pedostructure concept. Soil Sci. Soc. Am. J. 70, 494-502.

[8] BRonswiJk J.J.B. 1990. Shrinkage geometry of a heavy clay soil at various stresses. Soil Sci. Soc. Am. J., 54, 1500-1502.

[9] BRONSWIJK J.J.B. 1991. Relation between vertical soil movements and water-content changes in cracking clays. Soil Sci. Soc. Am. J. 55, 1220-1226.

[10] CABIDOCHE Y.M., RuY S. 2001. Field shrinkage curves of a a swelling clay soil: analysis of multiple structural swelling and shrinkage phases in the prisms of a vertisol. Austr. J. Soil Res. 39, 143-160.

[11] CAPPERT L.P., CASSIE F.W. 1971. The mechanism of engineering soils. $5^{\text {th }}$ ed. E.F.N.S.P.O.N; London.

[12] CAVAzZA L. 1981. Fisica del terreno agrario. UTET. Torino.

[13] Cavazza L., Guarnieri A., Patruno A., Lorenzini G., Cirillo E. 2005. Edotensiometro da laboratorio. Riv. di Ing. Agr., 4, 57-64.

[14] Cavazza L., Patruno A., Guarnieri A., Cirillo E., LORENZINI G. 2006. Specific volume variation in the upper layers of a typic vertisol. Riv di Ing. Agr. 37, 4, 35-42.

[15] Cavazza L. 2005. La struttura del suolo agrario. Riflessioni. Atti Accad. di Agricoltura di Torino, Vol. 147.

[16] CHERTKOV 2005. The shrinkage geometry factor of a soil layer. Soil Sci. Soc. Am. J. 69, 1671-1683.

[17] CNR-UNI 1003-74 e ISO 31-3-1992. Norme. Notiz. AIGR pag. 212, 1976.

[18] Crescimanno G., Provenzano G. 1999. Soil shrinkage characteristics curve in clay soils: measurements and prediction. Soil Sci. Soc. Am. J. 63, 25-32.

[19] Crescimanno G., DeSantis A. 2002. Il trasporto preferenziale di acqua e soluti in un terreno argilloso: indagine sperimentale. Riv. di Ing. Agr., 3, 41-48.

[20] Damiani P., Ciollaro G., Coppola A., ricostruzione dei profili di contenuto d'acqua in un suolo strutturato sottoposto a lavorazioni. Riv. Ing. Agr. (in corso di stampa).

[21] FRITTON D.D. 2006. Fitting uniaxial soil compression using initial bulk density, water content, and matric potential. Soil Sci. Soc. Am. J. 70, 1262-1271.

[22] Gardner E.A., Coughlan K.J., Smith G.D. 1990. Computation and use of bulk density in swelling soils. Soil Technology 3, 343-349. (Cit. da Cabidoche e Ruy).

[23] Garnier P., Rieu P., Boivin P., Vauclin M., Bavayé P. 1997. Determining the hydraulic properties of a swelling soil from a transient evaporation experiment. Soil Sci. Soc. Am. J. 61, 1555-1563.

[24] Garnier P., Perrier E., Angulo Jamarillo R., BaVAYÉ P. 1997. Numerical model of 3-dimensional anisotropic deformation and 1-dimensional water flow in swelling soil. Soil Sci. 162, 410-420.

[25] Groenevelt P.H., Bolt G.H. 1972. Water retention in soil. Soil Sci. 113, 238-245.

[26] Groenevelt P.H., Grant C.D., Semetsa S. 2001. A new procedure to determine soil water availability. Austr. J. Soil Res. 39, 577-598.

[27] HallaiRE V. 1984. Evolution of crack networks during a clay soil under grass and wheat crops. In: Bouma and Raats. Proc. Int. Soil Sci. Soc. Symp. Water and solute mov. In heavy clay soils. I.L.R.I, Wageningen. 
[28] KeEn B.A. 1931. The physical properties of the soil. Longman Green, London. (Cit. da Cabidoche e Ruy).

[29] Kim D.J., Angulo Jamarillo R., Vauclin M., Feyen J., CHOI S.I. 1999. Modeling of soil deformation and water flow in a swelling soil. Geoderma 92, 217-233.

[30] McGarry D.M., Malafant K.W.J. 1987. The analysis of volume change in unconfined units os soil. Soil Sci. Soc. Am. J. 51, $290,297$.

[31] MCInTYRE 1981. The properties and utilization of cracking clay soil. Rural Sciences, 5, 116-122.

[32] Mitchell A.R. van Genuchten Th. 1992. Shrinkage of bare and cultivated soil. Soil Sci. Soc. Am. J. 56, 1036-1042.

[33] OAdes J.M., Waters A.G. 1991. Aggregate Hierarchy of soils. Austr. J. Soil Res. 29, 815-828.

[34] Passioura J. 1981. The properties and utilization of cracking clay soils. Rural Science. 5, 116-121.

[35] Peng X., Horn R. 2005. Modeling soil shrinkage curve across a wide range of soil type. Soil sci. Soc. Am. J. 69, 584-592.

[36] Perroux K.M., Aromratama V., Boonyol S. 1974. Volume change and air-water relations of Chai Nat Soil; Thai. J. Agric. Sci. 7, 23-35 (Cit. by Mc Intyre).

[37] PhILIP J.R. 1971. Hydrology of swelling soils. In: Talsma T., Philip J.R. Salinity and water use. Mc Millan Press.

[38] PHILIP J.R. 1969. Hydrostatics and hydrodynamics in swelling soils. Water Resources Res. 5, 5, 1071-1077.

[39] Ross P., SMetTem K.R.J. 1993. Describing soil hydraulic properties with sums of simple functions. Soil Sci. Soc. Am. J. 57, 26-29.

[40] SMILEs D.E. 2000. Hydrology of swelling soils: a review. Austr. J. Soil Res. 38, 501-521.

[41] Sposito G. 1973. Volume changes in swelling soils. Soil Sci. 115, 315-320.

[42] STIRK G.B. 1954. Some aspects of soil shrinkage, the effect of cracking upon water entry into the soil. Austr. J. Agric. Res., 5, 279-290.

[43] Syers J.K., DeVRies F.P., Nyamudeza P. 2001. The sustainable management of vertisols. CABI Pub.

[44] Talsma T. 1977. Moisture profiles in swelling soils. Aust. J. Soil Res. (12), 71-75.

[45] Van Genuchten M.T. 1980. A closed-form equation for predicting the hydraulic conductivity of unsaturated soil. Soil Sci. Soc. Am. J. 44, 892-898.

[46] Van Genuchten M.T., Nielsen D.R. 1985. On describing and predicting the hydraulic properties of unsaturated soils. Ann. Geophys. 3, 615-628.

[47] Voltz M., Cabidoche Y.M. 1995. Non - uniform volume and water content changes in swelling clay soil: I. Theoretical analysis. Europ. J. Soil Sci. 46, 393-343.

[48] Yong R.N., Warkentin B.P. 1966. Introduction to soil behaviour. The Macmillan Co, New York.

[49] Yule D.F., RitchiE J.I. 1980. Soil shrinkage relationships in Texas Vertisol. II. Large cores. Soil Sci. Soc. Am. J. 44, 1291-1295.

Parole chiave: Suoli rigonfiabili; curve di contrazione e ritenzione dell'acqua; fessurazione dei suoli; conduttività idrica; consolidometri.

\section{SUMMARY}

\section{THE BEHAVIOUR OF AGRICULTURAL SWEL- LING SOILS}

The feature of single domains of a shrinkage curve
(ShC) for a swelling soil are recalled. The relationship between ShC and water retention curve (WRC) with special reference to the findings of Boiven, Garnier and Vauclin are shown and a short comment is given for the case of the basic domain. The contribution of the soil swelling process to the distribution of the total mechanical stress between solid and liquid phase according to a load factor is recalled for field conditions. Special attention is given to the cracking problems using the transformations of Bronswijk and Chertkov and after the theories of Yule and Ritchie and of Mc Intyre. Some considerations are expressed for the use of oedometers. The results of Cabidoche and Ruy on a cultivated vertisol open the possibility of a wider approach to these problems.

Key words: Swelling soils; Shrinkage and water retention curves; soil cracking; consolidometer.

\section{SIMBOLI}

\begin{tabular}{|c|c|c|}
\hline Simbalo & Definizinane & $\begin{array}{l}\text { Simbole } \\
\text { inglesse }\end{array}$ \\
\hline$V_{\text {ese }}$ & $\begin{array}{c}\text { Volume massice apparente } \\
\text { (dkl solido) }=\text { Pass }^{-1}\end{array}$ & $V_{\mathrm{s}}$ \\
\hline$w$ & awidis gravimetricar $\left(\mathrm{kg} \mathrm{kg}^{\prime}\right)$ & $\theta_{6}$ \\
\hline$\rho_{\text {sps }}$ & $\begin{array}{l}\text { massa volumicu apparente } \\
\text { (dici solidi) }\end{array}$ & $p_{n}$ \\
\hline$\rho_{i}$ & massa vaíwwica dei solidh & \\
\hline$P_{1}$ & morssa volumica defl' 'acyeng & \\
\hline$e$ & ragporto do' uwato & \\
\hline$\theta$ & napper fo of moinditi & \\
\hline
\end{tabular}

Simboli per le eymazioni di Cerikov

$$
\begin{aligned}
& \bar{V}_{0} \quad \text { valore insiole di } \bar{V} \\
& \bar{V}_{1} \quad V_{\text {map }} \text { delio siraio can fersure } \\
& \bar{V}_{1} \quad \text { come prima sec. Brarswije } \\
& \bar{V} \quad V_{\text {nam }} \text { di plasma sensa fersure } \\
& \bar{V}_{5} \quad V_{\operatorname{map}} d i \text { compione con fesaure } \\
& \text { a) weno rappario de's noto }
\end{aligned}
$$

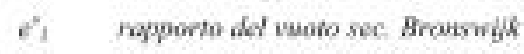

$$
\begin{aligned}
& \text { 1. } M \text { farnaricontrivi } \\
& \chi \quad \text { parawetro per l'eiasticitá del } \\
& \text { ierreve }=x(\mu)^{2}-\vec{V}_{1} / \bar{V}_{1} \\
& \mu \text { paramerino di clasticità ate' ternemo } \\
& \checkmark \quad \text { replowto di Poissen per it plaseia }
\end{aligned}
$$

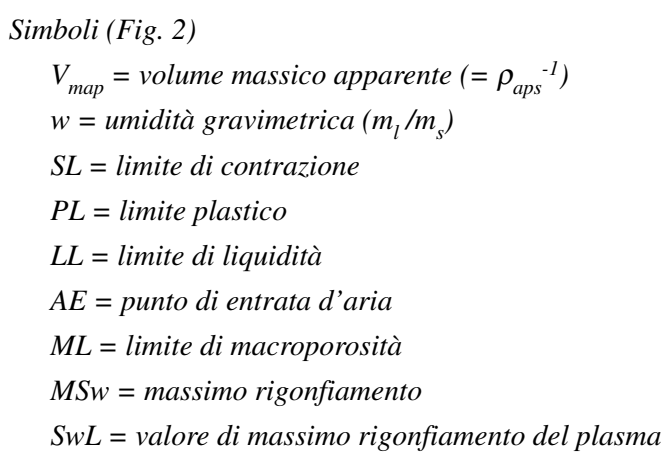

